\title{
On timing accuracy in observing radio impulses associated with Extensive Air Showers
}

\author{
Alain Lecacheux ${ }^{1}$, D. Charrier ${ }^{2}$, R. Dallier ${ }^{2}$, A. Escudie ${ }^{2}$, D. García-Fernández ${ }^{\dagger, 2}$, \\ L. Martin ${ }^{2}$ and B. Revenu' ${ }^{2}$. \\ ${ }^{l}$ CNRS-Observatoire de Paris, Meudon, France \\ ${ }^{2}$ Subatech, Institut Mines-Telecom Atlantique, CNRS, Universite de Nantes, Nantes, France \\ E-mail: alain. Iecacheux@obspm. fr
}

\begin{abstract}
A large subset of EAS radio observations by CODALEMA experiment in Nançay was used to measure the departure from a plane of the signal arrival times to the antennas. The study was done at two different scales in terms of range to the EAS axis. Below about $300 \mathrm{~m}$, by using a 10 antennas array triggered by neighbouring surface detectors, the average departure was found to be negligible (i.e. lower than the measurement incertitude of about 2-3 ns), leading to an equivalent "wave front curvature" radius larger than $30 \mathrm{~km}$. Above the $300 \mathrm{~m}$ range, and within the first kilometre, an extended array of several tens of self-triggered antennas was used. The measurements suggest the existence of significant time departures (of order $10 \mathrm{~ns}$ at $1 \mathrm{~km}$ range), which corresponds to an apparent curvature radius much larger than the atmosphere thickness. The main limitation of the method, in addition to the inferior time precision due to self-triggering, was the lack of accurate knowledge of corresponding EAS core locations, especially when those cores are located well outside the surface detector extent.
\end{abstract}




\section{Introduction}

Radio impulses associated with Extended Air Showers (EAS) produced in terrestrial atmosphere by High Energy Cosmic Rays (UHECR) of energy $10^{17} \mathrm{eV}$ and above, are now routinely observed by dedicated radio instruments on ground. Two basic, non-radiative processes [1] are able to produce significant, impulsive change in the electric field measured by a distant observer: i) a "charge excess" due to differential absorption of negatively and positively charged secondary particles along the EAS development; ii) a "charge displacement" of those particles, leading to a "transverse current" and its implied electric field by the Lorentz force exerted by Earth's magnetic field [2].

In both processes, two different versions of the resulting field can be produced, depending on whether the charge is moving slower of faster than the velocity of light in the medium [3]. In the first version, - the infra-luminous case -, only one instant in the charge's past history has a light cone which reaches any given location in space-time (e.g. an antenna). In the second version, - the supra-luminous case -, the field visibility is restricted to from inside the Čerenkov cone. Since the air index is variable with altitude and composition (in particular humidity), the two infra and supra luminous versions of each emission mechanism can coexist within a single shower observed by a spatially distributed antenna array.

Furthermore, in the aim to better explain radio observations, most of the recent numerical simulation models (e.g. CoREAS [4], ZHAireS [5], SELFAS2 [6]) do include an additional radiative term (acceleration), expressing the "time-variation" of both charges and transverse current.

In order to check and disentangle all those contributions, the real signals have to be carefully analysed. For instance, the accurate measurement of times of arrival on the individual antennas of a radio array is a way of sampling in space the EAS electric field distribution (i.e. the shape of the wave front in case of a purely radiative process), for comparison with corresponding model predictions.

In the following, a large subset of EAS radio observations by CODALEMA experiment in Nançay (France) is used to accurately measure the departure from a plane of the signal arrival times to the antennas.

\section{Observations and analysis}

\subsection{Instrumentation}

Three different CODALEMA sub-systems (Fig.1) have been used in this work (see [7] for a more detailed description of the CODALEMA experiment).

The surface detector (SD) is made of 13 scintillators covering a limited area of $400 \mathrm{~m} \times$ $400 \mathrm{~m}$, allowing unambiguous detection of high energy cosmic ray events. It provides a trigger signal sent to CA and to a dedicated GPS station for accurate time recording and off-line time coincidence with the stand-alone antenna stations (SA). Firing the trigger requires that five scintillators out of 13 can see a signal above the level corresponding to an energy detection threshold at $10^{15} \mathrm{eV}$.

The compact array (CA) is made of a cluster of 10 active antennas in dual linear horizontal polarisation, distributed over a square of about $150 \mathrm{~m} \times 150 \mathrm{~m}$ (24 to $146 \mathrm{~m}$ spacing). The 
elementary antenna is a dual inverted V, fat dipole, similar to the ones used in the Long Wavelength Array interferometer (LWA1) in USA [8], but equipped with LONAMOS wideband, low noise amplifiers [9]. The output voltages of each pair of dipoles are digitized by a $400 \mathrm{MHz}, 14$ bits ADC, then down converted to base band and added in quadrature, in order to provide 10 circularly polarized output signals without too much dependence on azimuth. Each $\mathrm{ADC}$, triggered by the surface detector (scintillators), runs for $6.5 \mu \mathrm{s}$, with a time resolution of $2.5 \mathrm{~ns}$ and a time (relative) accuracy much better than $1 \mathrm{~ns}$.

Fifty seven self-triggered standalone stations (SA) are scattered over roughly $1 \mathrm{~km}^{2}$ around the two former CODALEMA sub-systems. Each station is made of two crossed horizontal fat dipoles, fed to same LONAMOS low noise amplifiers as those used in the CA array. Same ADC boards are also used, but working with higher $(1 \mathrm{GHz})$ sampling rate. A multi-level triggering system has been developed to select narrow transients specific to air shower induced electric field and to reject signals produced by anthropic noise sources. An internal GPS clock ensures common time scale with SD and CA, with an accuracy better than $5 \mathrm{~ns}$.

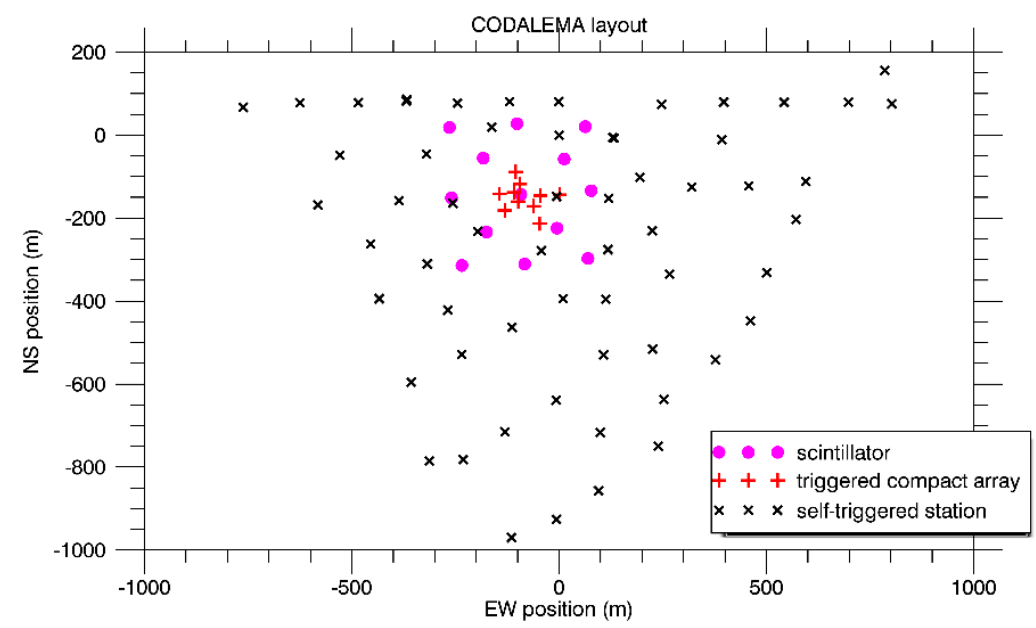

Figure 1: layout of the CODALEMA experiment

\subsection{Method}

Timing reconstruction of EAS radio signals is a special case of the well-studied TDoA (Time Difference of Arrival) problem, belonging to the general DoA (Direction of Arrival) problem when only signal timing is considered. Correctly solving this problem is conditioned by the accuracy of the sensor positions knowledge, the accuracy of the event dating and the relevance of the emitting source model.

Let $N$ sensors $\left\{\mathcal{A}_{j}\right\}_{j=1 . . N}$ located at positions $\vec{r}_{j}$ and measuring at times $t_{j}$ (or pseudoranges $p_{j}=c t_{j}$ ) the arrival of a perturbation propagating at the light velocity $c$ in direction $-\vec{u}$. Let $\vec{R}$ and $P$ the position and pseudo-range (time of arrival at ground) of the EAS radio core (by analogy with the EAS particles core). We can consider four models:

1) the perturbation source is at distance $\mathrm{C}^{-1}$ in direction $\overrightarrow{\mathrm{u}}$ :

$$
p_{j}=P-C^{-1}+\left\|C^{-1} \vec{u}-\vec{r}_{j}\right\|=P-C^{-1}\left(1-\sqrt{1-2 C \vec{u} \cdot \vec{r}_{j}+C^{2}\left\|\vec{r}_{j}\right\|^{2}}\right)
$$

which reduces (in far field limit $\left\|\overrightarrow{\mathrm{r}}_{j}\right\| \mathrm{C} \ll 1$ ) to: 


$$
p_{j} \approx P-\vec{u} \cdot \vec{r}_{j}+\frac{C}{2}\left\|\vec{u} \times \vec{r}_{j}\right\|^{2}
$$

2) the source is at infinite $(\mathrm{C}=0)$ :

$$
p_{j}=P_{\infty}-\vec{u}_{\infty} \cdot \vec{r}_{j}
$$

3) the isochrones are planar surfaces perpendicular to $\overrightarrow{\mathrm{u}}$ : same expression as in model 2

4) the isochrones are curved surfaces of revolution around the $\vec{u}$ axis:

$$
p_{j}=P-\vec{u} \cdot \vec{r}_{j}+\frac{C}{2} \mathcal{F}\left(\vec{u} \times\left(\vec{r}_{j}-\vec{R}\right)\right)
$$

where $\mathcal{F}$ is a function of the antenna distance to the EAS axis, satisfying:

$\mathcal{F}(0)=\mathcal{F}^{\prime}(0)=0$ and $\mathcal{F}^{\prime \prime}(0)=1$.

The latter expression (model 4) can summarize all the other models. Here, the parameter $C$ is the curvature of the isochrone at its intersection with the EAS axis (i.e. at the EAS core location).

It might be tempting to use this expression for building, from the $\left\{r_{j}, p_{j}\right\}$ measurements alone, a least squares estimation of the $P, \vec{u}, C, \vec{R}$ unknowns as well as of some additional parameters describing $\mathcal{F}$ (as, for instance, in [10]). Without entering further mathematical discussion (not possible because of the allowed length of these Proceedings), it should be clear that such a calculation would lead to solve an ill-conditioned problem and likely be not conclusive, since $\vec{R}$ and $\mathcal{F}$ are of the second order with respect to $P, \vec{u}, C$.

Therefore, we have adopted a simplified two steps procedure: the first step is a fit of the model 2 (or 3 ) to the $\left\{r_{j}, p_{j}\right\}$ measurements, providing the $P_{\infty}, \vec{u}_{\infty}$ solution and a set of residuals $\delta p_{j}$; the second step consists in a fit of model 4 to $\left\{r_{j}, \delta p_{j}\right\}$ by choosing $\mathcal{F}(d)=d^{2}$ (parabolic isochrone), yielding the system of equations:

$$
\left\{\delta p_{j}=\delta P-\delta \vec{u} \cdot \vec{r}_{j}+\frac{C}{2}\left\|\vec{u}_{\infty} \times \vec{r}_{j}\right\|^{2}\right\}_{j=1 . . N}
$$

in which $\delta P, \delta \vec{u}$ and $C$ are new unknowns, assumed to be of the same order of magnitude. Finally, the amount of curvature can be tested upon magnitude of the $\chi^{2}$ decrease and significance of the $\mathrm{C}$ parameter departure from 0 .

\subsection{Results}

All elements of the CODALEMA experiments were recently geolocalized with an

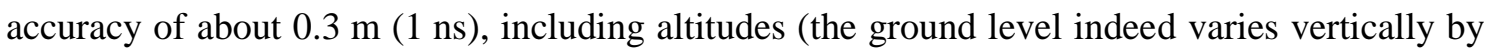
$\pm 8 \mathrm{~m}$ over the Nançay radioastronomy observatory area).

The efficiency of our DoA reconstruction was tested on several thousands of transient aircraft signals (obtained during hundreds of air flights over Nançay during the two last years) as well as on a large amount of recorded EAS radio events (more than 3000 at time of this writing). These two kinds of signal are by far the most frequent ones when looking at high elevation. Regarding the aircraft signals, the reconstruction (model 1 of section 2.2) was accurate enough for allowing identification of commercial flights and successful check of altitude, speed and heading of the aircraft.

From the statistical analyses above, the distribution of pseudo-range residuals, for the CA antenna array, was found to be unimodal, with a $1.5 \mathrm{~m}$ upper boundary and a peak (modal value) located at $0.75 \mathrm{~m}$, consistent with the used $2.5 \mathrm{~ns}$ sampling time (cf. Section 2.1). On the other hand, the pseudo-range residuals for SA antenna array display a similar distribution, but shifted towards higher values by a factor of about 3 . The mode of the distribution of time residuals is 
located at about $2.4 \mathrm{~m}$ (or $8 \mathrm{~ns}$ ), reflecting the loss of precision due to the GPS synchronisation scheme.

In order to answer the initial question about the shape of the EAS radio isochrones, we distinguished between two different ranges of distances to EAS axis.

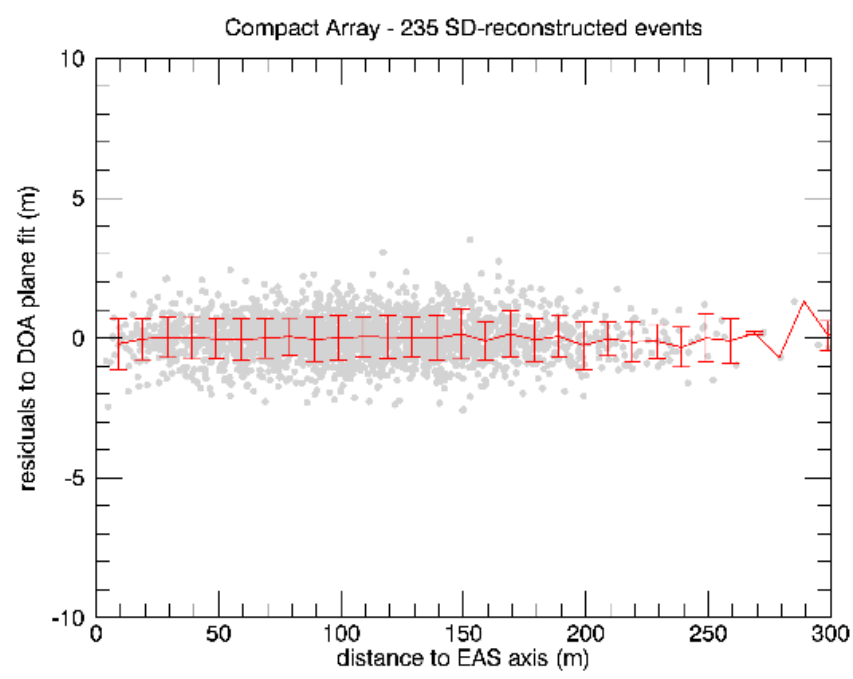

Figure 2: CA analysis of 235 EAS events. The $1 \sigma$ error bar is calculated for each $10 \mathrm{~m}$ range.

By using the CA instrument, we could explore the first $300 \mathrm{~m}$, by selecting 235 high quality events, well reconstructed (energy, core location, etc...) from the corresponding SD data. Results are displayed in Fig.2. No obvious curvature is visible, leading to a curvature radius, if any, larger than $30 \mathrm{~km}$.

By using the SA instrument, we could explore larger distances, up to about $900 \mathrm{~m}$, by using 34 more EAS events, also seen by the SD, but, unfortunately, badly reconstructed (as usually when the EAS core is outside the area containing the scintillators). Results are displayed in Fig.3. As for CA, but with a larger scattering, the distribution of residuals below $300 \mathrm{~m}$ appears to be flat around zero. Beyond $300 \mathrm{~m}$, a slight tendency for increase of the residuals is barely visible, reaching about $10 \mathrm{~ns}$ at $1000 \mathrm{~m}$, and leading to a curvature radius larger than 100 $\mathrm{km}$.

\section{Discussion and Conclusion}

Statistical analysis of the most recent CODALEMA data suggests that the isochrone surfaces of radio signals produced by Extensive Air Showers are weakly or not curved, with a radius of curvature larger than $\sim 30 \mathrm{~km}$, at the difference of the pancake of secondary particles which is known to show up a pronounced curvature (curvature radius of $\sim 8 \mathrm{~km}$ for a shower with energy of $\left.10^{18} \mathrm{eV}[11]\right)$ linked to the altitude of the maximum development $\left(\mathrm{X}_{\mathrm{MAX}}\right)$ of the shower.

Our result is in contradiction with previously published works $[10,12]$ which, on the contrary, found a more pronounced curvature of the radio front, with typical time departure of $\sim 20 \mathrm{~ns}(\sim 7 \mathrm{~m})$ at $300 \mathrm{~m}$ from EAS axis (cf. Fig.6 in [10] and compare with our Fig.2). Some further analysis is needed to resolve the contradiction.

Let us note that the existence of such a "radio curvature" is only legitimated by the assumption of a radiative part in the radio emission mechanism (implying that the radio antenna 
would be in the near field of an emitting distant source). In the opposite case, the relativistic effects are constraining impulses of electric field to occur when the observing antenna is just contained in a plane perpendicular to the particles propagation, implying a basically planar radio front.

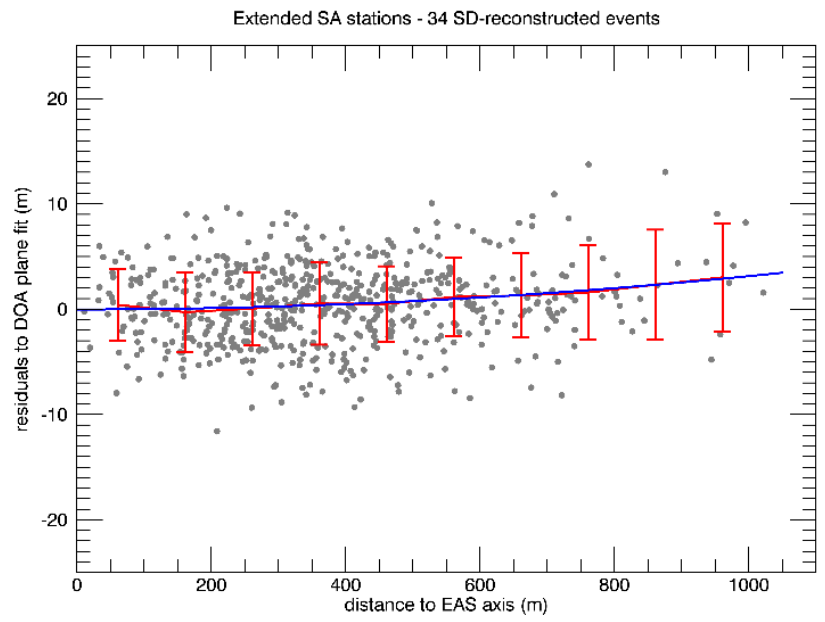

Figure 3: SA residual analysis for 34 external EAS events, for which core locations could only be estimated. The $1 \sigma$ error bar is calculated for each $100 \mathrm{~m}$ range. The blue curve is the best parabolic fit corresponding to the average curvature $C=0.0064 \pm 0.0006 \mathrm{~km}^{-1}$.

\section{Acknowledgmentss}

We thank the Région Pays de la Loire for its financial support of the Astro-particle group of Subatech and in particular for its contribution to the EXTASIS experiment. The Compact Array is a noticeable part of the support by the Observatoire de Paris to the CODALEMA experiment.

\section{References}

[1] O. Scholten, K. Werner and F. Rusydi, Astropart. Phys., 29, 94-103, (2008)

[2] F. D Kahn and I. Lerche, Proc. Roy. Soc. A, 289, 206, (1966)

[3] N. Meyer-Vernet, A. Lecacheux and D. Ardouin, Astron.Astrophys. 480, 15-25, (2008)

[4] T. Huege, M. Ludwig and C.W. James, AIP Conference Proceedings 1535, 128, (2013)

[5] J. Alvarez-Muniz, W.R. Carvalho and E. Zas, Astropart. Phys., 35, 325-341, (2012)

[6] V. Marin and B. Revenu, Astropart. Phys., 35, 733-741, (2012)

[7] B. Revenu et al., this conference.

[8] S.W. Ellingson et al., IEEE Trans. Ant. Prop., 61, 2540-2549, (2013)

[9] D. Charrier, Nuclear Instr. Meth., 662, S142-S145, (2012)

[10] A. Corstanje et al., Astropart. Phys., 61, 22-31, (2015)

[11] J. Linsley, J. Phys. G, 12, 51, (1986)

[12] W.D. Apel et al., J. Cosm. Astrop. Phys., 2014, sept. 2014, (2014) 